Chapman University

Chapman University Digital Commons

6-19-2019

Postoperative Pain: Factors and Tools to Improve Pain

Management in Children

Mai M. Makhlouf

Eric Robles Garibay

Brooke N. Jenkins

Zeev N. Kain

Michelle Fortier

Follow this and additional works at: https://digitalcommons.chapman.edu/psychology_articles

Part of the Other Psychiatry and Psychology Commons, Pediatrics Commons, Surgery Commons, and the Surgical Procedures, Operative Commons 


\section{Postoperative Pain: Factors and Tools to Improve Pain Management in Children}

\section{Comments}

This is a pre-copy-editing, author-produced PDF of an article accepted for publication in Pain Management, volume 9, issue 4, in 2019 following peer review. The definitive publisher-authenticated version is available online at https://doi.org/10.2217/pmt-2018-0079 .

\section{Copyright}

Future Medicine 


\section{Practice Points:}

Background:

Outpatient surgeries are becoming more common and therefore post-operative pain management is transitioned to parents and/ or guardians which has been correlated to suboptimal pain management.

\section{Recommendations for Pain Assessment:}

Because children express pain in variable ways depending on their age and developmental abilities, there are multiple ways to assess pain.

Cultural Factors and Pain

Cultural factors such as language, socioeconomic status, stoic beliefs, and biological differences (e.g. oxytocin) among groups may predispose parents and health care providers to treat child's pain suboptimally.

\section{Parent Pain Management of Child Pain}

Parental beliefs about medications, misconceptions about pain expression, and the opioid crisis modulation of parental attitudes have led parents to sub-optimally treat pain.

Tools and Technological Interventions Targeting Parental Management of Children's Pain

Tools and technological interventions have been developed to help parents assess and treat their child's pain.

\section{$\underline{\text { Best Practices }}$}

Child postoperative pain is complex and therefore its essential for healthcare providers and parents to work together to assess and treat pain considering cultural and developmental factors, along with accessible tools to further improve pain management.

\section{Summary:}

Outpatient surgery has made it increasingly common for parents to manage pain in the home setting. Studies have shown that parents often under treat pain, leaving children vulnerable to the negative side effects of suboptimal pain management. Multiple factors affect pain management like child's age and developmental stage, language, cultural values like stoicism, parental beliefs about medication, biological differences among groups, etc. Understanding all the factors involved can help healthcare providers and parents better understand pain and contribute to optimal pain management. Multiple tools and technological interventions have been created to help create a better understanding of pain and a holistic approach to care.

Keywords: Postoperative Pain, Parent pain management, opioids, Pain assessments, Pain management, Pediatric pain 


\section{Introduction:}

Although pain is an evolutionarily beneficial sensation that can promote avoidance behavior towards harmful stimulus,[1] it is also an unpleasant sensory and emotional experience[2] that can lead to a variety of potentially negative sequelae[3]. Thus, rather than attempting to eradicate the pain experience, it is necessary to develop strategies to manage pain in the most efficient and optimal way possible. Despite the fact that pain is a universal experience, there are a variety of factors that impact pain experience. These include, but are not limited to prior pain experience, age and development, ethnic and cultural background, and beliefs about pain [4], [5]. In addition, adults charged with managing children's pain, including healthcare providers and caregivers, are influenced by their own experiences with and beliefs about pain and may also be subject to variations in hospital practice, including the growing awareness of the opioid crisis in the context of pain management[6]. Thus, adequately managing pain in children is complex and evidence suggests that pain is overwhelmingly undertreated in children, particularly in the home setting[7].

Surgery is a common medical procedure that subjects children to pain, with over $85 \%$ of children experiencing pain postoperatively[8] [9] and 63\% experiencing clinically significant pain upon transition to home [8], [10]-[12]. Furthermore, the implications of postoperative pain are not limited to immediate consequences. Poorly managed postoperative pain could lead to unanticipated hospitalization[13], development of chronic pain[14], increased sensitivity to pain[15] and increased pain and anxiety during medical events hat take place later in life[16]. It is especially important to advocate for optimal pain management for children because consequences of untreated pain can lead to maladaptive behavioral changes, increased reliance on analgesics, and delayed postoperative recovery[16]. Children who have inadequate pain management may also be subject to a decrease in immune and neuroendocrine function later on in life[17]. Upon discharge following a surgical procedure, the burden of pain management is transferred from the health care provider to the parent, making it even more imperative that parents have knowledge in the most appropriate methods of assessing children's pain. Therefore, the purpose of this paper is to highlight pain management as a complex and multifaceted phenomena and to provide recommendations of factors that are necessary to consider in optimal pain management, including 
developmental stages, methods for pain assessment, cultural factors, parental practice of pain management at home and available tools.

\section{Pain Assessment}

It is important to note that children express pain in varying ways that are dependent on age and developmental stage[18]. Modes of pain expression include, but are not limited to, verbalizing, crying, and wailing. Behavior change may also be a strong indicator for parents to know that their child is in pain; in the case of older children, silence may be observed in conjunction with behavioral withdrawal when experiencing pain[19]. These differential expressions of pain based on developmental stages have implications for how parents assess children's pain severity. For parents and caregivers, pain is more easily assessed when the child is able to vocalize their pain experience; however frequency of verbalizing pain experience is highly dependent on the age and cognitive developmental stage of the child[20]. When children are unable to vocalize their pain verbally (e.g. infants, cognitively impaired and sedated children), assessment of pain is more difficult for both health care providers and parents because pain assessment is not as simple as asking the child about their discomfort.

Historically, self-report has been considered the gold standard for assessment of pain severity. However, this poses an obstacle for children who lack the ability to verbally report their pain due to age or developmental delay/cognitive impairment. Moreover, in recent years it has been proposed that relying on self-reported pain severity for intervention leads to an oversimplification of the pain experience as a whole[21] since children interpret pain scores in varying ways. That said, self-reported pain should still be the cornerstone of assessment in conjunction with contextual factors, clinical history and previous response to pain experiences [21], [22]. Several child-report pain measures have been developed to assess ratings of pain severity in preschool/school-aged children. These include the Wong-Baker FACES Scale (used for children 3 and older)[23] and the Faces Pain Scale-Revised (used for children aged 4 to 12)[24]. These scales allow children to point to their level of pain depicted through faces. Moreover, for children over the age of 7 the Visual Analog Scale is a tool that allows children to point to their level of pain on a line 100 mm long with one end representing "no pain" and the other representing "worst pain"[25]. 
There are a number of validated observational tools that are currently used for pain assessment in non- and pre-verbal children. For infants, the Neonatal Infant Pain Scale[26] and the Face, Legs, Activity, Cry, and Consolability (FLACC) scale (used for children under 4)[27] each provide observers with a checklist to assess pain-related behaviors. It is worth mentioning that recent findings have shown that the FLACC is most useful when used by non-experienced rather than experienced nurses[28]. Nonetheless, the FLACC remains the most widely used tool in the hospital setting[29]. Observational measures can also be utilized for children with cognitive disabilities. For example, the Non-communicating Children's Pain Checklist, an observational measure, and the FLACC have both been utilized to assess pain in children with disabilities[30]-[32].

The Parents Postoperative Pain Measure (PPPM), is a 15-item checklist containing behaviors that indicate pain (e.g. whining and holding the body area in pain) and behaviors that indicate recovery. PPPM has been an essential tool in research studies because it is the only validated measure for parental report of child pain[9]-[11], [33]. This tool is useful because it allows parents to correlate behavior with pain levels and lead to better assessment of pain medication post-surgery.

Physiological measures can also be helpful to take a more holistic approach when treating pain. Changes in blood pressure and heart rate can aid in determining the severity and impact of pain[34]. In addition, neuroimaging has bridged the gap between brain activity and pain experience by allowing researchers to measure changes in endogenous chemical pathways in response to painful experiences. There is no specific "pain center" in the brain, but rather six areas of the brain including the thalamus and anterior cingulate cortex that consistently respond to acute pain[35]. Physiological measures outlined above can be utilized in conjunction with self-assessment tools and observational measures to adequately treat pain.

Finally, both a bundled and hierarchical approach have been propositioned to assess pain holistically[21]. The bundled approach centers on the acronym "CARES" which stands for "Context, Assess Pain Expression, Risk Factors, Emotional Factors, and Socio-cultural factors". Each of these components are taken into consideration together to yield an accurate assessment for pain. In contrast, the hierarchical approach, advocated by the American Society for Pain Management in Nursing[36], brings the 
same factors as "CARES" into consideration, but with self-report being of most importance to careproviders when assessing pain[37]. Clinical judgment is a catalytic factor for effective evaluation in both of these two propositioned heuristic approaches which requests the need for a simplified yet multifaceted process in addressing pain management.

Table 1: Pain Assessment Approaches

\begin{tabular}{|c|c|c|}
\hline Approach & Tool/ Measure & Description \\
\hline \multirow[t]{4}{*}{ Healthcare Provider Report } & $\begin{array}{l}\text { Faces, Legs, Activity, Cry, } \\
\text { and Consolability (FLACC) } \\
\text { Scale[38] }\end{array}$ & $\begin{array}{l}\text { A validated observational tool used for } \\
\text { pain assessment in non- and pre-verbal } \\
\text { children, for ages } 4 \text { and under, and/or for } \\
\text { children with cognitive disabilities. } \\
\text { Observers select pain-related behaviors } \\
\text { from a checklist for a total score between } \\
0-10 . \text {. }\end{array}$ \\
\hline & $\begin{array}{l}\text { Non-Communicating } \\
\text { Children's Scale [39] }\end{array}$ & $\begin{array}{l}\text { An observational pain measure used for } \\
\text { children with cognitive disabilities. This } \\
\text { measure prompts health care professionals } \\
\text { to award a score of } 0-3 \text { ( } 0 \text { being not often } \\
\text { and } 3 \text { being very often) to assess how often } \\
\text { vocal, social, facial, activity and } \\
\text { physiological indicators of pain occur. The } \\
\text { score is summed to yield an overall pain } \\
\text { score. }\end{array}$ \\
\hline & $\begin{array}{l}\text { Context, Assess Pain } \\
\text { Expression, Risk Factors, } \\
\text { Emotional Factors, and } \\
\text { Socio-cultural factors } \\
\text { (CARES) [36] }\end{array}$ & $\begin{array}{l}\text { A bundled approach that values the } \\
\text { context, pain expression assessment, risk } \\
\text { factors, emotional factors, and socio- } \\
\text { cultural factors to yield an accurate } \\
\text { assessment for pain. }\end{array}$ \\
\hline & *Hierarchical Approach [36] & $\begin{array}{l}\text { A hierarchical approach, similar to } \\
\text { CARES in its components, ranks self- } \\
\text { report as the highest factor when assessing } \\
\text { pain in children. }\end{array}$ \\
\hline Parent-Report & $\begin{array}{l}\text { Parents Postoperative Pain } \\
\text { Measure (PPPM) [33] }\end{array}$ & $\begin{array}{l}\text { A } 15 \text {-item checklist designed for children } \\
\text { ages } 7-12 \text { years containing behaviors that } \\
\text { indicate pain and recovery (e.g. whining), } \\
\text { allowing parents to assess their child's } \\
\text { pain. It is the only validated measure for } \\
\text { parental report in research studies. Scores } \\
\text { range from } 0-15 ;>6=\text { clinically } \\
\text { significant pain. }\end{array}$ \\
\hline Child-Report & Wong Baker Faces Scale [40] & $\begin{array}{l}\text { A self-report scale designed for children } \\
\text { ages } 3 \text { and older. Children point to their } \\
\text { level of pain depicted through } 6 \text { faces } \\
\text { differing in their facial expression and }\end{array}$ \\
\hline
\end{tabular}




\begin{tabular}{|c|c|c|}
\hline & & $\begin{array}{l}\text { corresponding numerical value ranging } \\
\text { from } 0-10 \text {. }\end{array}$ \\
\hline & $\begin{array}{l}\text { Faces Pain Scale-Revised } \\
\text { [41] }\end{array}$ & $\begin{array}{l}\text { A self-report scale used for children ages } 4 \\
\text { and older that allows children to point to } \\
\text { their level of pain depicted through } 6 \text { faces } \\
\text { differing in their facial expression and } \\
\text { corresponding numerical values ranging } \\
\text { from } 0 \text { to } 10 \text {. }\end{array}$ \\
\hline & $\begin{array}{l}\text { Visual Analog Scale (VAS) } \\
\text { [42] }\end{array}$ & $\begin{array}{l}\text { A self-report scale that allows children to } \\
\text { select their level of pain based on a } 100 \mathrm{~mm} \\
\text { horizontal line with anchors at each end } \\
\text { such that } 0=\text { no pain experienced and } 100 \\
=\text { worst pain experienced. This scale is } \\
\text { used for children over the age of } 7 \text {. }\end{array}$ \\
\hline \multirow[t]{2}{*}{ Physiological Report } & Blood Pressure [34] & $\begin{array}{l}\text { Changes in blood pressure and heart rate } \\
\text { may indicate severity of pain experienced } \\
\text { through an objective lens }\end{array}$ \\
\hline & $\begin{array}{l}\text { Functional Magnetic } \\
\text { Resonance Imaging } \\
\text { (MRI)[35] }\end{array}$ & $\begin{array}{l}\text { Neuroimaging can allow healthcare } \\
\text { providers to measure changes in chemical } \\
\text { pathways to obtain an objective measure of } \\
\text { pain severity. }\end{array}$ \\
\hline
\end{tabular}

\section{Cultural Factors and Pain}

There are many cultural factors such as language, socioeconomic status (SES), stoicism, and biological differences (levels of oxytocin) among groups that can impact pain experience[43]-[46] Language is an important cultural mediator that can affect how parents manage pain. Language fluency can impact health literacy and ability to adequately convey/comprehend recommendations for pain management that may lead to inadequate pain management (e.g. low medication adherence)[43]. Moreover, it has been observed that Spanish-speaking parents may be more likely to experience misconceptions regarding analgesic use for children (e.g. fear of medication side effects) compared to English-speaking parents, ultimately contributing to providing fewer doses of analgesics after children's surgery [47]. English-speaking Hispanic parents have been found to be less likely to use analgesics for their child's pain compared to English-speaking White parents and Spanish-speaking Hispanic parents[48]. A possible reason for this observation may be that English-speaking Hispanic parents are bicultural, which places parents between two possible modes of treating pain, traditional with respect to their Hispanic culture and more 
conventional methods utilized in Western medicine[49]. Thus, it is important to consider how acculturation may impact children's pain management practice in the home setting.

Cultural values such as stoicism, enduring pain without expression or complaint, in Hispanic culture may play a role in pain expression and therefore affect pain management[45]. It is important to note, however, that stoicism is a multi-dimensional moderator that also includes sociocultural factors (e.g., employment and community[50]. When faced with pain, individuals from a Hispanic culture often face this adversity in a stoic manner believing that lower pain expression is a reflection of personal strength and pride[45]. In a cohort of Hispanic adults undergoing cancer pain management, stoicism was observed to be a common response to pain[45]. Given that stoicism may be common in Hispanic culture, stoicism expressed by children may lead parents to believe that their child is not experiencing pain and therefore administer less medication for their pain[49]. Stoicism could be an explanatory variable in a study showing that Hispanic children who underwent outpatient tonsillectomy and adenoidectomy received suboptimal pain management[10]. Further research is necessary to show this correlation. Because pain expression is often gauged by observing child behavior or verbal expression[20], children who experience stoicism may be at a disadvantage. Therefore, paying attention to cultural factors like stoicism will be useful for both parents and health care providers.

Biological processes may have an influence on how different ethnicities perceive pain as well as experience it. For example, oxytocin, commonly known as the good-feeling neuromodulator, has been shown to inhibit pain sensation[51]. Oxytocin is produced and released from the hypothalamus, having downstream effects in the spinal cord and blood stream, inhibiting pain sensation by repressing pain fibers with oxytocin; therefore, preventing nociceptive signals from being perceived (limited to mechanical and thermal sensitivities in inflammatory responses)[51]. Historically, it has been shown that African Americans have a tendency to experience more pain than non-Hispanic whites[46]. In a study comparing oxytocin levels in African American women vs white women when undergoing a pain task, African Americans had lower levels of oxytocin[46]. Moreover, this result also reflects the fact that African American women had lower pain tolerance to the experimental stressors[46]. This research shows that there 
may be biological processes that differ, affecting how different racial/ethnic groups may experience pain sensitivity.

Although cultural and socioeconomic factors may be difficult to parse out, distinguishable characteristics of each are important in determining a child's pain experience. Several studies have shown the association between socioeconomic status (SES) and the experience of pain. Specifically, it has been observed that individuals living in less affluent socio-economic areas experience a higher prevalence of pain[44]. On an individual level, lower SES based on education[52] and income[53] has been correlated with a higher frequency and intensity of pain. Families with lower SES may have more limited resources and lower health literacy that ultimately impacts children's pain experience and management.

\section{Parent Management of Child Pain}

Because there has been a shift to conducting surgeries for children on an outpatient basis[54], parents are left with the responsibility to manage their child's pain at home. It has been observed, however, that parents often provide suboptimal pain management. For example, children undergoing tonsillectomy and adenoidectomy (T\&A), a common pediatric surgical procedure, were given fewer doses than the optimal number parents are instructed to administer [10]. Of the doses provided, $70 \%$ were considered sub therapeutic, leaving pain untreated[48]. It has also been observed that parents may modify provider pain management instructions. More specifically, parents may provide alternative pain medications (e.g., overthe-counter instead of prescription) or provide medications less frequently over a longer period of time[55][59]. Parents have been shown to display misconceptions such as medications should be given only if their child is displaying intolerable pain or less medication is better[60]. It is also possible that parents have fears of possible addiction. In addition, parents often believe that children cry when they are in pain and therefore may not provide medication when their child is quiet, but may still be experiencing pain[60]. Moreover, parents may hold ideas that children complain and express their pain for other reasons such as wanting attention[60]. Thus, parents who are not able to detect pain will likely be unable to optimally treat pain adequately. In addition, these misconceptions have been linked to the provision of fewer analgesics to children after surgery[48]. 
It is difficult to directly pinpoint the causes of suboptimal pain management at home by parents, because in addition to parental attitudes, child responsiveness and systemic factors influence medication administration and therefore, pain management. For instance, a child may refuse to take medications resulting in inadequate doses. Systemic factors such as difficulty obtaining medications may consequently affect medication administration [61]. With such a wide range of contributing factors, it is clear that pain management is difficult and therefore, should be viewed with a holistic lens. In order to appropriately consider a multifaceted approach, it is necessary to understand familial context and educate on the risks of suboptimal pain management.

The opioid epidemic may further influence parental attitudes towards administrating proper analgesic dosages to their children in the home setting. This epidemic has affected children and adolescents; hospitalizations caused by opioid poisonings have nearly doubled over a span of 15 years from 1997 to 2012[62]. More recently, hospitalizations for opioid poisoning increased from 6.7 per 10,000 admissions in 2004 to 10.9 per 10,000 admissions in 2015[63]. Although episodes of opioid poisoning are greater in older adolescents (15-19 years of age), the greatest jump in opioid poisonings occurred in children aged 1 to 4 years[62]. These trends indicate that pain management is not only about preventing suboptimal pain management by under treating pain, but also preventing medication overuse. Because of increased incidence of use and abuse of opioid medications, parental attitudes affected by the opioid crises may influence proper dosage given post-surgery, whether that be under treatment or over treatment.

Although there is substantial evidence that parents tend to provide suboptimal treatment in the home setting, a recent study has shown that parents may be better as estimated children's pain compared to physicians.[64] Moreover, a recent qualitative study by Rabbitts and colleagues demonstrated that parents felt unprepared for managing pain and regaining functional abilities after their child's surgery.[65] This study highlighted the need for more intervention preparing children and families for surgery and managing recovery at home. Any efforts to optimize postoperative pain management in children in the home setting must therefore include appropriate psychosocial intervention to address the needs of children and caregivers 
in managing the demands of pain management and recovery at home as well as understanding appropriate pain assessment and dosing in order to provide adequate medication and/or non-pharmaceutical treatment.

\section{Tools and Technological Interventions Targeting Parental Management of Children's Pain}

To address analgesic safety and improve analgesic decision-making, Scenario-Tailored Opioid Messaging Program (STOMP) was developed[66]. STOMP is a non-clinical intervention that was given to parents while in the surgical or general pediatric waiting rooms. STOMP consists of a scenario-tailored message feedback based on interactive decision-making exercises, where parents are presented with scenarios and make decisions accordingly. After each scenario, feedback is presented assessing the risks associated with their decisions and ways to reduce risks for future similar scenarios. Parents who underwent the intervention became significantly risk avoidant (e.g., withheld medication to children displaying excessive sedation) compared to baseline and they were more likely to provide medications in situations where drug administration was safe (e.g. lack of excessive sedation)[66]. This intervention improved decision-making competency and shows promising ideas that can improve parental pain management. STOMP emphasizes the significance of providing pain management information in various ways like using an interactive scenario program. The implications of this program can be incorporated into home interventions for caregivers treating a child that has undergone surgery to improve pain management.

Moreover, there are a variety of non-pharmacological strategies for pain management that can be used by parents in the home setting. These include behavioral strategies, such as distraction and imagery, and physical intervention including heat and ice. However, ensuring parents are well-equipped to use such strategies can be difficult in a busy clinical setting. Thus, parent-focused interventions have been developed relying on technology to arm parents with the necessary pain management skills for children after surgery. For example, our research team has created a Web-based Tailored Intervention for Preparation of Parents and Children for Outpatient Surgery (WebTIPS), a web-based behavioral preoperative preparation program for children and parents that provides information and coping skills training before, during, and after an operation[67]. WebTIPS is tailored to the individual child considering anxiety, temperament, and surgery type. It is also tailored to parents by taking account for variable coping style, pain management attitudes, 
baseline anxiety and other measures. In a small-scale trial, this program proved effective in reducing both parent's and children's preoperative anxiety along with decreased emergence delirium in children,[67] factors that are known to be associated with postoperative pain. In an age in which web use is common, tools like these can be used as proactive measures to alleviate concerns before operation procedures and provide postoperative pain management education. WebTIPS contains modules focused specifically on managing pain in the home setting and a current multisite trial funded by the National Institutes of Health is underway to examine the impact of WebTIPS on children's pain after surgery.

Our team has also created Pain Buddy, an interactive, animated application that uses an avatar to guide children through an application to assess and manage pain. This application allows children to report their daily pain and symptoms, allows healthcare professionals to monitor symptoms and intervene to manage pain in real time, and provides cognitive and behavioral skills training as means for coping with pain. To increase user adherence, an incentive system has been implemented to motivate children to continue using the application[68]. Pain Buddy was developed for children undergoing cancer treatment, but has wide ranging applicability to manage pain in children in the home setting associated with a variety of medical conditions and procedures, and is a means of equipping both parents and children with the needed knowledge and skills to optimize pain management at home.

Future Directions

Ensuring proper assessment of children's postoperative pain at home is catalytic in adequately treating pain. Standardizing children's postoperative pain management can be a critical first step, although ensuring the translation of such protocols into practice is crucial. In addition, educating parents and children in proper pain management is vital, given the shift to outpatient care that has occurred across medical settings. Technology is highly prominent in societal use, therefore using it to educate parents on validated pain scales and the various ways a child expresses pain can deem beneficial when children are in pain but are unable to verbally express their discomfort. Furthermore, technological interventions, such as WebTIPs, are promising tools to optimize home pain management after surgery to both combat misconceptions about pain and ensure adequate knowledge in pain assessment and management in children. Use of technology 
also provides a wealth of opportunities to incorporate multimodal assessment that may be helpful in assessment of pain in children, given variations in pain based on surgical experience, age, ethnicity, temperament and behavioral patterns. When used in conjunction with proper analgesic administration, implementing technological applications that provide behavioral pain management strategies such as distraction, imagery and relaxation can reduce children's acute pain. Using this multimodal approach of combining pharmacological and nonpharmacological pain management strategies, child's pain can be managed more efficiently. In addition to educating parents in the home setting, assumptions cannot be made in the hospital setting about parental understanding of the appropriate analgesic dosages to give to their child. Because analgesic administration is variable depending on a child's age and weight, healthcare providers must ensure that parents understand how to administer analgesics to their child correctly and mend any concerns parents have for administrating these medications to their children. When answering concerns, healthcare providers must be aware of cultural factors that can influence how parents treat their child's pain; it is vital that instructions for analgesic use are given to parents in their language of choice.

\section{Conclusion}

Pain is common among children and today children's surgeries have increasingly been conducted in an outpatient basis, leaving the responsibility of pain management in the hands of parents. Moreover, providing optimal pain management is imperative given poorly managed pain in children after surgery has negative consequences such as delayed postoperative recovery or overdose in cases of medication overuse. There are many factors that affect how parents handle pain management in the home setting, including child development, culture, and parental beliefs/experience that together affect children's pain management. Assessment and treatment of pain is difficult for both healthcare providers and caregivers because of differing developmental stages of children and the broad spectrum of disabilities that exist in the young population. Today, many tools for pain assessment have been standardized in child healthcare. In addition to child developmental factors affecting pain management, there are various cultural factors that may moderate the effects of pain management, specifically, language. Non-English speakers may be at a disadvantage when understanding healthcare provider instructions and recommendations for optimal pain 
management in the home setting. In addition, cultural values may impact beliefs about pain expression and treatment. Similarly, parental attitudes towards opioid usage may be heavily influenced by the opioid crises. Although limited literature is available on this topic, it is important to understand the influence of healthcare issues in the world and how they may create misconceptions. Accordingly, treating pain is a multimodal process, therefore, proper pain assessment and education are catalytic in a child's overall wellbeing. Technology has shaped the world in many ways, but its impact on educating parents in the healthcare field is of utmost importance to emphasize. Within the next 5-10 years, we expect that technological innovations will allow for online sources and tablet applications to help parents and providers improve pain management. With the overwhelming evidence showcasing the advantages of personalized medicine, these tools are expected to become more personalized to each patient based on factors such as culture, age, ethnicity and cognitive development[69]. These tools will better prepare children and their families for surgical procedures and guide parents and providers in improving the post-surgery experience of children. With the current rise in technological innovations it is expected to see further healthcare technological interventions that leads to better outcomes for children of different ages and of different cultural backgrounds in order to help eliminate health disparities. 


\section{References}

[1] V. Bonavita and R. De Simone, "Pain as an evolutionary necessity," Neurol. Sci., vol. 32, no. SUPPL. 1, pp. 61-66, 2011.

[2] IASP Task Force on Taxonomy, Classification of Chronic Pain, 2nd Ed. Seattle: IASP Press, 2004.

[3] J. T. Pate et al., "Children' s Health Care Childhood Medical Experience and Temperament as Predictors of Adult Functioning in Medical Situations Childhood Medical Experience and Temperament as Predictors of Adult Functioning in Medical Situations," vol. 9615, 2010.

[4] M. M. Free, "Cross-cultural conceptions of pain and pain control.," Proc. (Bayl. Univ. Med. Cent)., vol. 15, no. 2, pp. 143-5, 2002.

[5] M. D. Good, P. E. Brodwin, and G. B. J, Pain as Human Experience: An Anthropological Perspective. University of California Press, 1994.

[6] and M. National Academies of Sciences Engineering and H. and M. D. B. on H. S. P. C. on P. M. and R. S. to A. P. O. Abuse, Pain Management and the Opioid Epidemic. National Academic Press, 2017.

[7] M. A. Fortier, L. S. Sender, and Z. N. Kain, "Management of Pediatric Oncology Pain in the Home Setting,” J. Pediatr. Hematol. Oncol., vol. 33, no. 4, pp. 249-250, 2011.

[8] M. A. Fortier, J. Chou, E. L. Maurer, and Z. N. Kain, “Acute to chronic postoperative pain in children: Preliminary findings," J. Pediatr. Surg., vol. 46, no. 9, pp. 1700-1705, 2011.

[9] Z. N. Kain, L. C. Mayes, A. Caldwell-Andrews, D. E. Karas, and B. C. McClain, "Preoperative anxiety, postoperative pain, and behavioral recovery in young children undergoing surgery.," Pediatrics, vol. 118, no. 2, pp. 651-658, 2006.

[10] M. A. Fortier, J. E. MacLaren, S. R. Martin, D. Perret-Karimi, and Z. N. Kain, "Pediatric Pain After Ambulatory Surgery: Where's the Medication?," Pediatrics, vol. 124, no. 4, pp. e588-e595, 2009.

[11] D. W. Stewart, P. G. Ragg, S. Sheppard, and G. A. Chalkiadis, "The severity and duration of 
postoperative pain and analgesia requirements in children after tonsillectomy, orchidopexy, or inguinal hernia repair," Paediatr. Anaesth., vol. 22, no. 2, pp. 136-143, 2012.

[12] G. Williams et al., "The prevalence of pain at home and its consequences in children following two types of short stay surgery: a multicenter observational cohort study.," Paediatr. Anaesth., vol. 25, no. 12, pp. 1254-63, Dec. 2015.

[13] K. C. Coley, B. A. Williams, S. V Dapos, C. Chen, and R. B. Smith, "Retrospective Evaluation of Readmissions After Same Day Surgery and Associated Costs,” vol. 8180, no. 02, pp. 349-353, 2002.

[14] F. M. Perkins and H. Kehlet, "Chronic pain as an outcome of surgery: A review of predictive factors," Anesth, vol. 93, no. 4, pp. 1123-1133, 2000.

[15] A. Taddio, J. Katz, A. L. Ilersich, and G. Koren, "Effect of neonatal circumcision on pain response during subsequent routine vaccination," Lancet, vol. 349, no. 9052, pp. 599-603, 1997.

[16] J. Pate, R. Blount, L. Cohen, and A. Smith, "Childhood medical experience and temperament as predictors of adult functioning in medical situations," Child. Heal. Care, vol. 25, pp. 281-298, 1996.

[17] G. G. Page, "Are There Long-Term Consequences of Pain in Newborn or Very Young Infants?," J. Perinat. Educ., vol. 13, no. 3, pp. 10-17, 2004.

[18] R. Srouji, S. Ratnapalan, and S. Schneeweiss, "Pain in Children: Assessment and Nonpharmacological Management," Int. J. Pediatr., vol. 2010, pp. 1-11, 2010.

[19] E. R. Katz, J. Kellerman, and S. E. Siegel, "Behavioral distress in children with cancer undergoing medical procedures: developmental considerations.," J. Consult. Clin. Psychol., vol. 48, no. 3, pp. $356-365,1980$.

[20] A. Dubois, S. Bringuier, X. Capdevilla, and R. Pry, "Vocal and Verbal Expression of Postoperative Pain in Preschoolers," Pain Manag. Nurs., vol. 9, no. 4, pp. 160-165, 2008.

[21] A. Twycross, T. Voepel-Lewis, C. Vincent, L. S. Franck, and C. L. Von Baeyer, "A Debate on the Proposition that Self-report is the Gold Standard in Assessment of Pediatric Pain Intensity," Clin. 
J. Pain, vol. 31, no. 8, pp. 707-712, 2015.

[22] C. L. Von Baeyer, "Children's self-report of pain intensity: What we know, where we are headed," Pain Res. Manag., vol. 14, no. 1, pp. 39-45, 2009.

[23] M. J. Hockenberry and D. Wilson, Wong's Essentials of Pediatric Nursing, 8th ed. St. Louis, MO: Mosby, 2009.

[24] D. Bieri, R. A. Reeve, G. D. Champion, L. Addicoat, and J. B. Ziegler, "The faces pain scale for the self-assessment of the severity of pain experienced by children: Development, initial validation, and preliminary investigation for ratio scale properties," Pain, vol. 41, no. 2, pp. 139$150,1990$.

[25] K. H. Todd, K. G. Funk, J. P. Funk, and R. Bonacci, "Clinical significance of reported changes in pain severity,” Ann. Emerg. Med., vol. 27, no. 4, pp. 485-489, 1996.

[26] J. Lawrence, D. Alcock, P. McGrawth, J. Kay, S. MacMurray, and C. Dulberg, "The development of a tool to assess neonatal pain.," Neonatal Netw. NN, vol. 12, no. 6, pp. 59-66, 1993.

[27] R. Manworren and L. Hynan, "Clinical Validation of FLACC: Preverbal Patient Pain Scale." pp. 140-146, 2003.

[28] J. Shen et al., "Evaluation of nurse accuracy in rating procedural pain among pediatric burn patients using the Face, Legs, Activity, Cry, Consolability (FLACC) Scale,” Burns, vol. 43, no. 1, pp. 114-120, 2017.

[29] T. Voepel-Lewis, J. Zanotti, J. A. Dammeyer, and S. Merkel, "Reliability and validity of the face, legs, activity, cry, consolability behavioral tool in assessing acute pain in critically ill patients," Am. J. Crit. Care, vol. 19, no. 1, pp. 55-61, 2010.

[30] R. Nader, T. F. Oberlander, C. T. Chambers, and K. D. Craig, "Expression of Pain in Children With Autism," Clin. J. Pain, vol. 20, no. 2, pp. 88-97, 2004.

[31] K. L. Hadden and C. L. Von Baeyer, "Pain in children with cerebral palsy: Common triggers and expressive behaviors," Pain, vol. 99, no. 1-2, pp. 281-288, 2002.

[32] P. J. McGrath, C. Romus, C. Canfield, M. A. Campbell, and A. Hennigar, "Behaviours caregivers 
use to determine pain in non-verbal, cognitively impaired individuals," Dev. Med. Child Neurol., vol. 40, pp. 340-343, 1998.

[33] C. T. Chambers, G. J. Reid, P. J. McGrath, and G. A. Finley, "Development and preliminary validation of a postoperative pain measure for parents," Pain, 1996.

[34] R. Cowen, M. K. Stasiowska, H. Laycock, and C. Bantel, “Assessing pain objectively: The use of physiological markers," Anaesthesia, vol. 70, no. 7, pp. 828-847, 2015.

[35] D. L. Morton, J. S. Sandhu, and A. K. P. Jones, "Brain imaging of pain: State of the art," J. Pain Res., vol. 9, pp. 613-624, 2016.

[36] K. Herr et al., "Pain Assessment in the Nonverbal Patient: Position Statement with Clinical Practice Recommendations," Pain Manag. Nurs., vol. 7, no. 2, pp. 44-52, 2006.

[37] C. Pasero and M. McCaffery, Pain Assessment and Pharmacologic Management. St. Louis: Elsevier Mosby, 2011.

[38] S. I. Merkel, T. Voepel-Lewis, J. R. Shayevitz, and S. Malviya, "The FLACC: a behavioral scale for scoring postoperative pain in young children," Pediatr. Nurs., vol. 23, no. 3, pp. 293-297, 1997.

[39] L. M. Breau, G. A. Finley, P. J. McGrath, and C. S. Camfield, "Validation of the Noncommunicating Children's Pain Checklist-Postoperative Version,” Anesthesiology, vol. 96, no. 3, pp. 528-535, 2002.

[40] D. L. Wong and C. M. Baker, "Pain in children: comparison of assessment scales," Pediatr. Nurs., vol. 14 , no. 1 , pp. $9-17,1988$.

[41] D. Bieri, R. A. Reeve, G. D. Champion, L. Addicoat, and J. B. Ziegler, "The Faces Pain Scale for the self-assessment of the severity of pain experienced by children: development, initial validation, and preliminary investigation for ratio scale properties," Pain, vol. 41, no. 2, pp. 139-150, 1990.

[42] A. M. Carlsson, "Assessment of chronic pain. I. Aspects of the reliability and validity of the visual analogue scale.," Pain, vol. 16, no. 1, pp. 87-101, May 1983.

[43] Y. Hsu, C. Mao, and M. Wey, “Antihypertensive medication adherence among elderly Chinese 
Americans," J Transcult Nurs, vol. 21, no. 4, pp. 297-305, 2010.

[44] T. E. Dorner, J. Muckenhuber, W. J. Stronegger, É. Rsky, B. Gustorff, and W. Freidl, “The impact of socio-economic status on pain and the perception of disability due to pain," Eur. J. Pain, vol. 15, no. 1, pp. 103-109, 2011.

[45] G. Juarez, B. Ferrell, and T. Borneman, "Influence of culture on cancer pain management in Hispanic patients," Cancer Pract., vol. 6, no. 5, pp. 262-269, 1998.

[46] K. M. Grewen, K. C. Light, B. Mechlin, and S. S. Girdler, "Ethnicity is associated with alterations in oxytocin relationships to pain sensitivity in women," Ethn. Heal., vol. 13, no. 3, pp. 219-241, 2008.

[47] M. L. Batista, M. A. Fortier, E. L. Maurer, E. Tan, H. C. Huszti, and Z. N. Kain, "Exploring the impact of cultural background on parental perceptions of children's pain," Child. Heal. Care, vol. 41, pp. 97-110, 2012.

[48] R. Y. Zisk Rony, M. A. Fortier, J. M. Chorney, D. Perret, and Z. N. Kain, "Parental postoperative pain management: attitudes, assessment, and management," Pediatrics, vol. 125, no. 6, pp. e13728,2010 .

[49] E. Olshansky, R. Zender, Z. N. Kain, A. Rosales, J. Guadarrama, and M. A. Fortier, "Hispanic parents' experiences of the process of caring for a child undergoing routine surgery: A focus on pain and pain management," J. Spec. Pediatr. Nurs., vol. 20, no. 3, pp. 165-177, 2015.

[50] A. Moore, J. Grime, P. Campbell, and J. Richardson, “Troubling stoicism: Sociocultural influences and applications to health and illness behaviour," Heal. An Interdiscip. J. Soc. Study Heal. Illn. Med., vol. 17, no. 2, pp. 159-173, 2013.

[51] M. Eliava et al., "A New Population of Parvocellular Oxytocin Neurons Controlling Magnocellular Neuron Activity and Inflammatory Pain Processing,” Neuron, vol. 89, no. 6, pp. 1291-1304, 2016.

[52] K. Hagen, L. Vatten, L. J. Stovner, J. Zwart, S. Krokstad, and G. Bovim, “Low socio-economic status is associated with increased risk of frequent headache: a prospective study of 22718 adults 
in Norway.," Cephalalgia, vol. 22, no. 8, pp. 672-9, 2002.

[53] C. R. Green and T. Hart-Johnson, "The association between race and neighborhood socioeconomic status in younger black and white adults with chronic pain," J. Pain, vol. 13, no. 2, pp. 176-186, 2012.

[54] K. A. Cullen, M. J. Hall, and A. Golosinskiy, "Ambulatory surgery in the United States, 2006," Natl Heal. Stat Rep., no. 11, pp. 1-25, 2009.

[55] G. A. Finley, P. J. McGrath, S. P. Forward, G. McNeill, P. Fitzgerald, and Anonymous, "Parents' management of children's pain following 'minor' surgery," Pain, vol. 64, no. 1, pp. 83-87, 1996.

[56] J. P. H. Hamers and H. H. Abu-Saad, "Children's pain at home following (adeno)tonsillectomy," Eur. J. Pain, 2002.

[57] P. Kankkunen, K. Vehvilainen-Julkunen, A. M. Pietila, H. Kokki, P. Halonen, and Anonymous, "Parents' perceptions and use of analgesics at home after children's day surgery," Paediatr. Anaesth., vol. 13, pp. 132-140, 2003.

[58] H. M. Munro, S. Malviya, G. R. Lauder, T. Voepel-Lewis, A. R. Tait, and Anonymous, "Pain relief in children following outpatient surgery," J. Clin. Anesth., vol. 11, pp. 187-191, 1999.

[59] F. F. Warnock and J. Lander, "Pain progression, intensity and outcomes following tonsillectomy," Pain, vol. 75, no. 1, pp. 37-45, 1998.

[60] R. Y. Zisk, M. Grey, J. E. MacLaren, and Z. N. Kain, "Exploring sociodemographic and personality characteristic predictors of parental pain perceptions," Anesth. Analg., vol. 104, no. 4, pp. 790-798, 2007.

[61] J. E. MacLaren and Z. N. Kain, "Prevalence and predictors of significant sleep disturbances in children undergoing ambulatory tonsillectomy and adenoidectomy," J Pediatr Psychol, vol. 33, no. 3, pp. 248-257, 2008.

[62] J. R. Gaither, J. M. Leventhal, S. A. Ryan, and D. R. Camenga, "National trends in hospitalizations for opioid poisonings among children and adolescents, 1997 to 2012," JAMA Pediatr., vol. 170, no. 12, pp. 1195-1201, 2016. 
[63] J. Kane, J. D. Colvin, A. Bartlett, and M. Hall, "Opioid-Related Critical Care Resource Use in US Children's Hospitals," Pediatrics, vol. 141, no. 4, 2018.

[64] C. Brudvik, S.-D. Moutte, V. Baste, and T. Morken, “A comparison of pain assessment by physicians, parents and children in an outpatient setting.," Emerg. Med. J., vol. 34, no. 3, pp. 138 144, Mar. 2017.

[65] J. A. Rabbitts et al., "Long-Term Pain and Recovery After Major Pediatric Surgery: A Qualitative Study With Teens, Parents, and Perioperative Care Providers.," J. Pain, vol. 18, no. 7, pp. 778786, Jul. 2017.

[66] T. Voepel-Lewis et al., "Effect of a Scenario-tailored Opioid Messaging Program on Parents' Risk Perceptions and Opioid Decision-making," Clin. J. Pain, vol. 34, no. 6, pp. 497-504, 2018.

[67] M. A. Fortier et al., "Web-Based Tailored Intervention for Preparation of Parents and Children for Outpatient Surgery (WebTIPS): Formative Evaluation and Randomized Controlled Trial," Anesth. Analg., vol. 120, no. 4, pp. 915-922, 2015.

[68] M. A. Fortier, W. W. Chung, A. Martinez, S. Gago-Masague, and L. Sender, "Pain buddy: A novel use of m-health in the management of children's cancer pain.," Comput. Biol. Med., vol. 76, pp. 202-14, Sep. 2016.

[69] F. R. Vogenberg, C. I. Barash, and M. Pursel, "Personalized Medicine Part 1 : Evolution and Development into Theranostics," vol. 35, no. 10, 2010. 